\title{
兽 Confinement Leads to Spatially Oscillatory Collective Motion of Chiral Active Matter
}

A ctive matter consisting of self-propelling units. e.g. bacterial solutions and self-driven colloids, is an important class of soft matter systems. Due to its inherent out-of-equilibrium nature, active matter often exhibits exotic behaviors and has potential applications in microdevices and smart materials. Currenty. exploration of chiral active matter composed of selfspinning objects, breaking both parity and time-reversal symmetries, is an exciting and a rapidly developing area. I particularly interesting phenomenon of chiral active matter is the emergence of a spontaneous unidirectional collective edge flow when in confinement. The edge flow is proven to be even topologically protected. with potentials for robust material transport. This unique feature has generally been discussed in the context of incompressible structureless and homogeneous fluid. Extensive studies have revealed that the spinnerboundary interactions play an essential role in the emergence of the edge flow. I lowever, besides the steric interactions. confinement can abo cause a spatially nonuniform density distribution due to particle-position correlations. The density inhomogeneity is ubiquitous in confined interacting many-body systems and its effect on the collective behavior remains elusive.

In a new study, entitled "Oscillating collective motion of active rotors in confinement". published in Proceedings of the National Academy of Sciences of the United States of America on May 19 . a research group led by Prof. YANG Mingcheng and Prof. YE Fangfu from the Institute of Physics (IOP). Chinese Academy of Sciences (CAS), collaborating with Prof. ZIIENG Ning's group from the Beijing Institute of Technology, has made great progress in understanding the collective motion of confined chiral active matter (Fig. 1).

By combining theoretical calculation, numerical simulation. and experimental observation. the researchers investigate the effects of the density inhomogeneity on the emergent collective behavior in confined active spinners. They find a striking spatially oscillating collective motion of the chiral
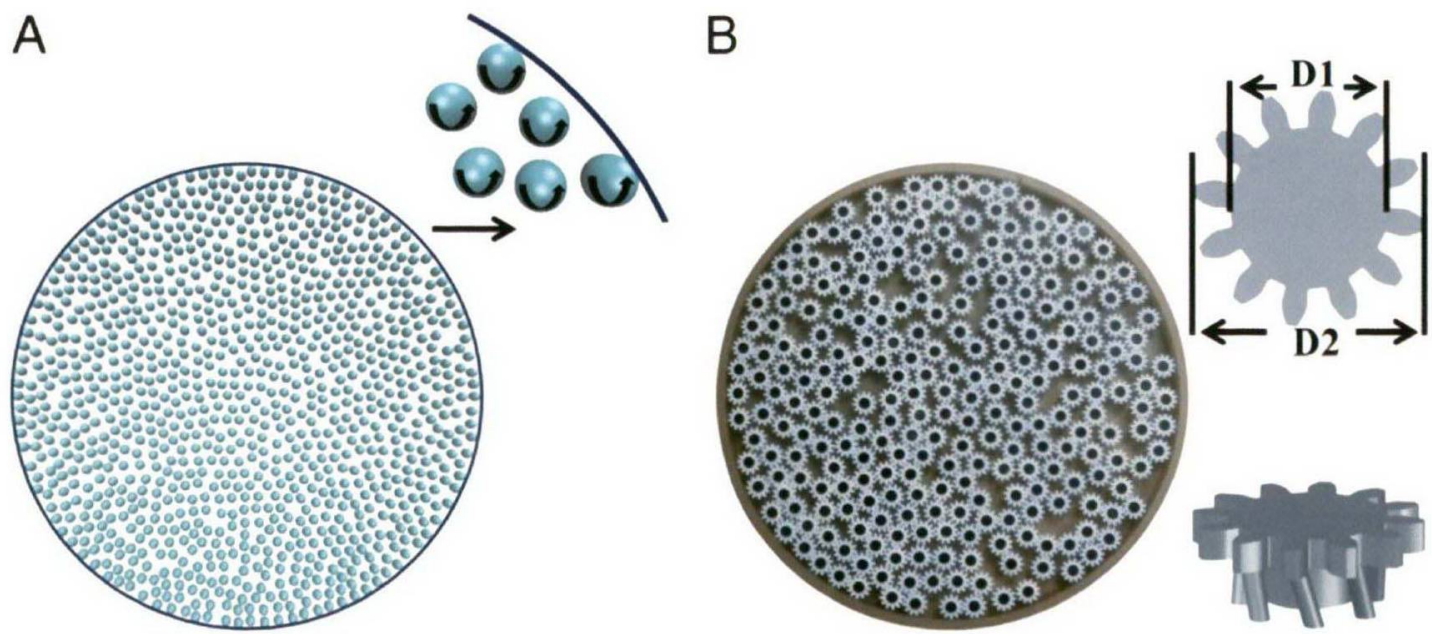

Fig. 1 Schematic diagram of simulation and experiment systems. (Image by IOP) 

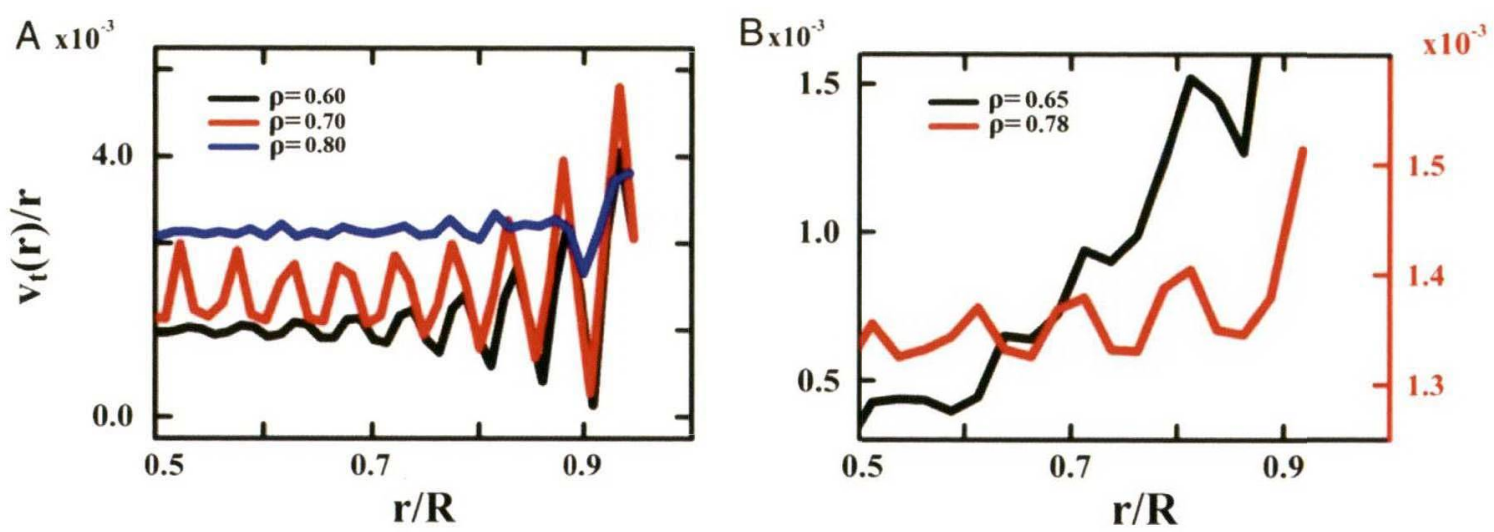

Fig. 2 Different collective motion modes from simulation and experiment. (Image by IOP)

active matter along the confinement boundary (Fig. 2). Microscopically, this oscillatory collective motion is generated by a position-dependent frictional stress originating from the nonuniform distribution of spinners. The researchers further show that the collective behavior experiences three different modes: viscous, viscoelastic, and elastic, as the system density varies. The structural origins of the transitions between the different modes are well identified by the percolation of solid-like regions or the occurrence of defect-induced particle rearrangement (Fig. 3).

These findings significantly advance our understanding of collective motion of chiral active matter in confinement, and highlight the importance of compressibility and inhomogeneity in the emergent collective behavior of active systems.

This study was supported by the National Natural Science Foundation of China, the Key Research Program of Frontier Sciences of the Chinese Academy of Sciences, and the K. C. Wong Education Foundation.

(IOP)

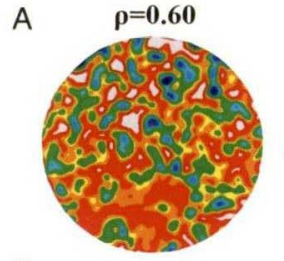

B
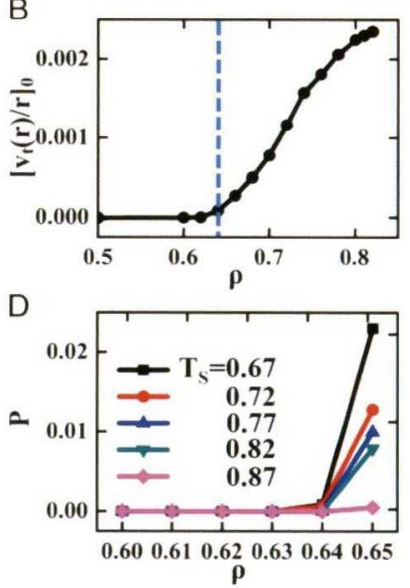

$\boldsymbol{\rho}=\mathbf{0 . 7 0}$
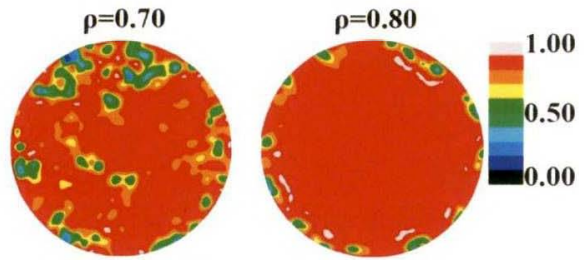

C

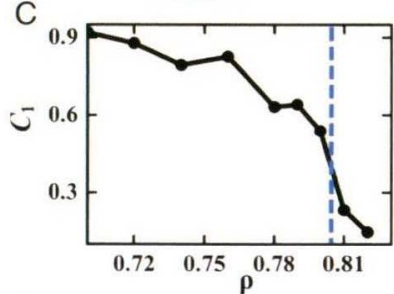

E

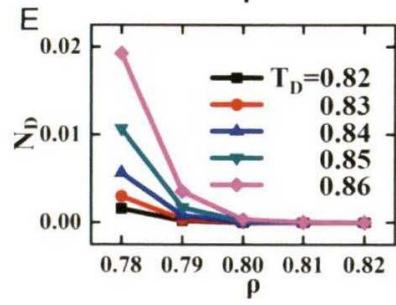

Fig. 3 Structure origins of different motion modes identified by local crystalline order, probabilities of percolation, and mean numbers of defects. (Image by IOP)

Contact:

YANG Mingcheng

Institute of Physics, CAS

Email: mcyang@iphy.ac.cn 